\title{
3D digitalization and micro-analysis for cultural heritage research: the case study of the bishop's head of Casa Romei Museum in Ferrara.
}

\author{
ANTONELLO AQUILANO ${ }^{1}$, MS. MARIA NICOLI, PHD $^{1}$, \\ ANDREA QUINTINO SARDO ${ }^{2}$ AND ELENA \\ MARROCCHINO $^{1}$ \\ ${ }^{1}$ University of Ferrara \\ ${ }^{2}$ Direzione Regionale Musei Emilia-Romagna/Bologna \\ Presenting Author: nclmra@unife.it
}

The unexpected COVID-19 pandemic has had an unprecedented impact on human life and forced the governments in Italy, and many other countries, to enforce the total closure of all non-essential structures and activity, including museums [1]. In this context, museums, unable to open their doors to visiting public, have had to take the only option of spreading culture and knowledge through online means, implementing a heavy process of digitization of the artistic heritage. Among the various methodologies adopted, 3D modelling represents one of the most effective methods for disclosure purposes in the field of cultural heritage.

In this study, an automatic digital photogrammetry software, 3DF Zephyr, has been used to obtain a 3D model of a stone artefact preserved inside the Casa Romei Museum in Ferrara. This study has been focused on the remaining part of an ancient stone statue representing a bishop: the head. The head is well defined with lots of particulars in the front part, while the back part is not well delineated. In the back part there is a hole where it is still possible to recognize the remains of a metal bar, this suggests that probably in the past the statue was fixed to a wall in a niche.

Several studies assert that a multidisciplinary approach is the best practice for a reliable evaluation of the conservation state of cultural artefacts, alsoin foresight of possible restoring intervention on them. For this reason, small fragments, both of natural stone material and metal, have been sampled from the back part of the bishop's head to perform chemical-mineralogical investigations. Raman micro-spectroscopy and Scanning Electron Microscope (SEM) analyses helped to determine the composition of the natural stone material used for the statue and to characterize the metal found in the cavity and its alteration products. Therefore, this study aims to combine digital technologies and analytical tools in order to support and improve the conservation and the communication of museum heritage.

[1] Agostino, Arnaboldi \& Lampis (2020) Museum Management and Curatorship, 35 (4), 362-372, DOI: 10.1080/09647775.2020.1790029 